\title{
Use of triamcinolone-soaked absorbable gelatin foam (gelfoam) as a treatment for recurrent sinonasal polyposis after FESS in poor candidates for revision surgery
}

Mohamed M. ElSheikh(i)

\begin{abstract}
Background: Chronic rhinosinusitis with nasal polyps recurs frequently after functional endoscopic sinus surgery (FESS), with resultant poor quality of life with persistence of nasal obstruction, sinus aches, and discharge as well anosmia and dysgysia; revision FESS is usually the treatment of choice referred to relief patients' suffering, yet it is not always the case, patients preference as well as fitness for general hypotensive anesthesia, patients age, cardiopulmonary status, and concurrent bronchial asthma might render unfit for an elective procedure. The aim of this study was to evaluate the efficacy of triamcinolone-soaked absorbable gelatin foam (gelfoam) as a treatment for recurrent sinonasal polyposis after FESS in poor candidates for revision surgery.
\end{abstract}

Results: Thirty patients suffering from recurrent sinonasal polyposis after single or multiple FESS procedures were selected; all patients were subjected to intranasal application of gelfoam soaked with triamcinolone as a conservative medical office procedure using triamcinolone-soaked absorbable gelatin foam (gelfoam) weekly for a period of 8 weeks followed by intranasal steroid nasal spray for maintenance and as anti-recurrence measure. Patients were followed up for 4 months postintervention; Meltzer Clinical Scoring System was used to analyze the patients' pre- and post-intervention via office endoscopy ( $\left(0^{\circ} 4-\mathrm{mm}\right.$ Hopkins endoscope). All patients tolerated the procedure well. The study group showed no statistical significance pre-intervention which comprised 17 (56.7\%) patients with grade 4 sinonasal polyposis extent and 13 (43.3\%) patients with grade 3 sinonasal polyposis extent $(P=0.795)$, denoting no difference between patients pending treatment option. Post-treatment, both groups showed a significant reduction in the extent of polyposis $(P<0.001)$ where posttreatment showed 21 (70\%) patients with grades 0 no polyps and 9 (30\%) patients with grade 1 sinonasal polyposis extent.

Conclusion: Triamcinolone-soaked gelfoam packing is an effective and safe method for managing recurrent sinonasal polyposis after FESS. Intervention contributed to a successful result, decreased the need for surgery in poor candidates for hypotensive anesthesia, and could be used as a sole treatment of recurrent sinonasal polypsis in elderly patients whose general condition could not tolerate invasive procedures.

Keywords: Recurrent sinonasalpolyposis, Triamcinolone, Gelfoam application

Correspondence: elsheikh.mohamed@hotmail.com

Department of Otorhinolaryngology, H\&N Surgery, Faculty of Medicine,

Helwan University, Cairo 11371, Egypt

Springer Open

๑ The Author(s). 2021 Open Access This article is licensed under a Creative Commons Attribution 4.0 International License, which permits use, sharing, adaptation, distribution and reproduction in any medium or format, as long as you give appropriate credit to the original author(s) and the source, provide a link to the Creative Commons licence, and indicate if changes were made. The images or other third party material in this article are included in the article's Creative Commons licence, unless indicated otherwise in a credit line to the material. If material is not included in the article's Creative Commons licence and your intended use is not permitted by statutory regulation or exceeds the permitted use, you will need to obtain permission directly from the copyright holder. To view a copy of this licence, visit http://creativecommons.org/licenses/by/4.0/. 


\section{Background}

Chronic rhinosinusitis with nasal polyps recurs frequently after functional endoscopic sinus surgery (FESS), with resultant poor quality of life with the persistence of nasal obstruction, sinus aches, and discharge as well anosmia and dysgysia; revision FESS is usually the treatment of choice referred to relief patients' suffering, yet it is not always the case, patients preference is an important issue to take into consideration in planning treatment for such condition with the general global thinking of avoiding invasive operative intervention with its complications and absenteeism from work; patient prefers conservative procedures or minimally invasive procedures to attain cure.

Another issue faced by the rhinologic surgeon is the possibility and fitness for general hypotensive anesthesia with muscle relaxant as certain comorbidities might be faced as regards patients age, cardiopulmonary status, concurrent bronchial asthma, and chest allergy which could lead to the situation in which fitness for operation might be delayed, postponed, or even judged as being unfit for an elective procedure.

One of the most common causes of nasal obstruction is allergic sinonasal polyposis; a variety of medical and surgical treatments has been introduced for treating such a condition. Yet, frequent recurrences and frustration still yield to the physician caring for these patients. Recurrence secondary to allergic hypersensitivity reactions remains the major obstacle, although the relationship between sinonasal polyposis (SNP), allergy, and genetic-hereditary factors has yet to be clarified [1].

Sinonasal polyposis (SNP) is a chronic inflammatory disorder of the nasal cavity and paranasal sinus mucosal membranes that typically affects patients bilaterally in the form of a benign edematous mass (polyps), extending from the paranasal sinuses to the nasal cavity. The etiology of SNP remains uncertain, and the precise prevalence is not well known; factors or associated conditions include smoking, allergy, asthma, fungal sensitivity, nonsteroidal anti-inflammatory drug (NSAID) intolerance, and genetic factors $[2,3]$.

Diagnosis of SNP is based on the presence of major symptoms (nasal obstruction, altered smell, anterior and/or posterior rhinorrhea, and pain or facial pressure) and minor symptoms (sore throat, dysphonia, cough, malaise, fever, dental pain, halitosis or pain/discomfort in the ears); then, polyposis is confirmed through endoscopic evaluation and imaging [4]. Corticosteroids are the first-line treatment for chronic rhinosinusitis with polyposis, according to the most recent European and North American consensus documents. Approximately $10 \%$ of all patients undergoing endoscopic surgery show a poor response to surgical treatment and concomitant medical therapy $[5,6]$.

\section{Aim of the work}

The aim of this work was to study the effect of using intranasal application of gelfoam soaked with triamcinolone as a conservative medical office procedure on patients suffering from recurrent sinonasal polyposis after single or multiple FESS procedures in poor candidates for revision surgery and anesthesia fitness as regards outcome in nasal polyp size using office-based endoscopy and improvement in nasal obstruction.

\section{Methods}

Thirty patients suffering from recurrent sinonasal polyposis after single or multiple FESS procedures were selected; all patients were subjected to intranasal application of gelfoam soaked with triamcinolone as a conservative medical office procedure using triamcinolone-soaked absorbable gelatin foam (gelfoam) weekly for a period of 8 weeks (total 8 office base endoscopic application) into the middle meatus or in cases where the middle turbinate was jeopardized during previous surgical procedures into the superior meatus, followed by intranasal steroid nasal spray for maintenance and as anti-recurrence measure.

Patients were followed at 4 months post-intervention; Meltzer Clinical Scoring System [7] was used to analyze the patients' pre- and post-intervention via office endoscopy ( $0^{\circ} 4$-mm Hopkins endoscope).

This study is a prospective cohort study on 30 patients with recurrent sinonasal polyposis after single or multiple FESS procedures followed up at at Helwan University (Badr teaching hospital) ENT outpatient department between November 2019 and August 2020.

\section{Inclusion criteria}

The following are the inclusion criteria:

- Patients suffering from persistent nasal blockage due to recurrent sinonasal polyposis.

- Both sexes were included.

- Age ranged from 50 to 85 years of age.

- Diagnosis of recurrent sinonasal polyposis was diagnosed clinically via endoscopic examination (Hopkins $0^{\circ}$ 4-mm sinuscope) as well as CT PNS coronal cut bone window.

- Patients having chronic medical or physical disabilities such as diabetes mellitus, hypertension, or hepatitis $C$ virus infection were included in the study as these factors intervened with fitness for general hypotensive anesthesia.

\section{Exclusion criteria}

The following are the exclusion criteria:

1. Patients whose imaging studies revealed any form of acute rhinosinusitis or chronic sinusitis or septal 
deviation that could interfere with the result of the intervention

2. Patients with a systemic disease that could cause polyposis such as the following:

(a) Immotile cilia syndrome

(b) Cystic fibrosis

All patients were informed of the study protocol, were consented for the treatment options, and were subjected to a detailed history taking and rhinological history for the duration of nasal symptoms as well previous FESS operations.

\section{Ethics and consent to participate}

Subjects who agreed to participate in the study were asked to sign a written informed consent. All procedures contributing to this work comply with Good Clinical Practice Guidelines and the ethical principles of the Helwan University Research ethical committee (ENREC) guidelines on human experimentation and with the Helsinki Declaration of 1975, as revised in 2008. The protocol has been revised and approved by the Institution Review Board (IRB no. 5432).

Patients were then examined by means of a $4-\mathrm{mm} 0^{\circ}$ sinuscope (Hopkins) and visualized the extent of sinonasal polyps according to the Meltzer Clinical Scoring System (Fig. 1). The previous FESS details were noted and recorded as regards middle meatal antrostomy size, level of ethmoidectomy, sphenoidotomy, and frontal recess clearance if done.

All data were collected at 4 months post-treatment, the local effect of the intervention was studied using the same method, and data were statistically analyzed.

\section{Statistical methods}

Data management and analysis were performed using Statistical Package for Social Sciences (SPSS) vs. 23. Age was summarized as means and standard deviations and ranges, as appropriate. Categorical data were summarized as numbers and percentages. Age was explored for
Table 1 Summary of epidemiological data of the study group

\begin{tabular}{lll}
\hline & \multicolumn{2}{l}{ Study group } \\
\cline { 2 - 3 } & Mean & Range \\
\hline Age & $63 \pm 10.3$ & $50-85$ \\
Females & $13(43.3 \%)$ & \\
Males & $17(56.7 \%)$ & \\
\hline
\end{tabular}

normality using the Kolmogorov-Smirnov test and Shapiro-Wilk test. The test showed that age was normally distributed. Comparisons between pre-intervention and post-intervention were done using Student's $t$ test. Chisquare or Fisher's tests were used to compare with respect to the categorical data, as appropriate. All $P$ values are two-sided. $P$ values $<0.05$ were considered significant.

\section{Results}

The study group's mean age was $63 \pm 10.3$ years (range 50 to 85 years) comprising 13 females (43.3\%) and 17 males $(56.7 \%)$ with no statistical significance $(P=0.849)$ (Table 1).

\section{Effects of treatment}

All patients tolerated the procedure well (Table 2). The study group showed no statistical significance preintervention which comprised 17 (56.7\%) patients with grade 4 sinonasal polyposis extent and $13(43.3 \%)$ patients with grade 3 sinonasal polyposis extent $(P=0.795)$, denoting no difference between patients pending treatment option.

Post-treatment, both groups showed a significant reduction in the extent of polyposis $(P<0.001)$ where posttreatment showed $21(70 \%)$ patients with grade 0 no polyps and 9 (30\%) patients with grade 1 sinonasal polyposis extent (Fig. 2).

\section{Discussion}

Intranasal steroid applications are useful for sinonasal polypsosis. These medications are labeled for the

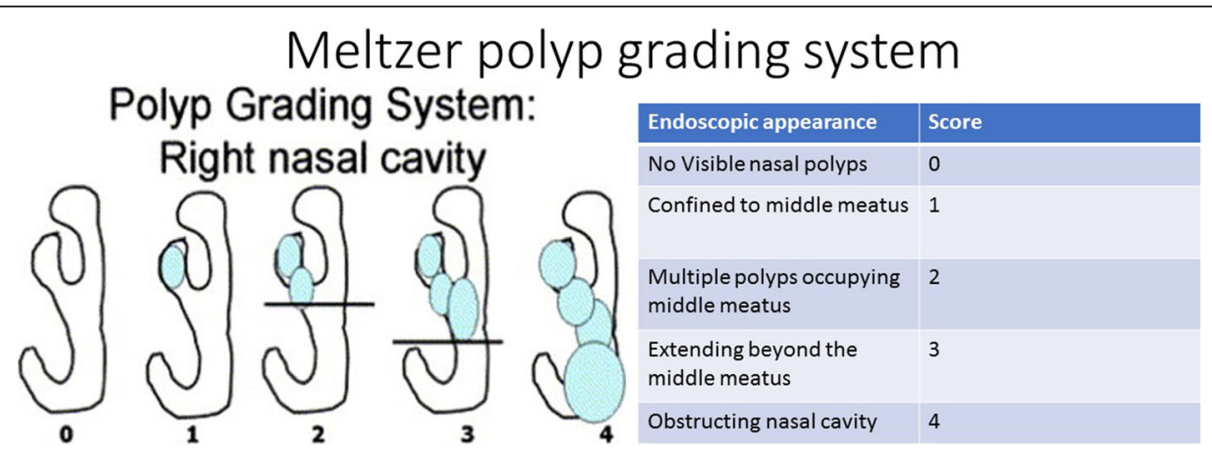

Fig. 1 The Meltzer Clinical Scoring System [7] 
Table 2 Summary of effects of treatment pre- and post-intervention in the study group at 4 months post-intervention

\begin{tabular}{lllll}
\hline & & Number & Percent & P value \\
\hline Extent of polyposis pre-treatment & Grade 3 & 13 & $43.3 \%$ & $56.7 \%$ \\
& Grade 4 & 17 & $70.0 \%$ & 0.795 \\
Extent of polyposis post-treatment & Grade 0 & 21 & $30.0 \%$ & $<0.001$ \\
\hline
\end{tabular}

management of allergic rhinitis but, like all steroids, also have nonspecific anti-inflammatory effects. Intranasal steroids may require continuous use for any significant benefits. Topical corticosteroids reduce blood flow and inhibit vascular permeability. The mechanisms for these actions include a reduction in the cyclo-oxygenase metabolites that maintain vascular beds, inhibition of phospholipase A2, and the subsequent formation of leukotrienes and platelet-activating factor (PAF); inhibition of the release of endothelial-derived relaxing factor; production of vasocortin (which reduces permeability); and enhancement of vasospasm by alpha adrenergic stimulation [8].

Surgical therapy is usually the case for symptomatic patients with persistent and/or recurrent sinonasal polyposis who are not responding to medical management.

In the current study, a prospective cohort analysis on 30 patients compared the extent of polyposis according to the Meltzer Clinical Scoring System after subjecting all patients to 8 weekly sessions of triamcinolone-soaked gelfoam. The study group showed no statistical significance pre-intervention which comprised $17(56.7 \%)$ patients with grade 4 sinonasal polyposis extent and $13(43.3 \%)$ patients with grade 3 sinonasal polyposis extent $(P=0.795)$, denoting no difference between patients pending treatment option. Post-treatment, both groups showed a significant reduction in the extent of polyposis $(P<$ $0.001)$ where post-treatment showed 21 (70\%) patients with grade 0 no polyps and $9(30 \%)$ patients with grade 1 sinonasal polyposis extent.
Actually, to date, no studies in the literature compared the treatment option as the current study, yet a study performed by Ryu et al. [9] on a total of 35 patients showed that endoscopic scores were significantly improved after triamcinolone-soaked gelfoam packing, and the effects were maintained at 3-month follow-up $(1.85 \pm 0.61$ vs. $0.82 \pm 0.77, P<0.001)$. In contrast to my study, they applied a single session of triamcinolone-soaked gelfoam packing and followed their result till 3 months post-intervention; in my study, the analytical stage was attained at 4 months post-intervention after 8 weekly applications and provided similar scores.

In another study by Pletcher and Goldberg [10], the SNOT-20 nasal symptom questionnaire and videoendoscopymodified POSE score were utilized to grade patient improvement; they performed statistically significant results in comparing patient improvement after 1 week and 1 month posttreatment (2.44 versus $1.65, P<0.05$, and 2.44 versus $1.36, P$ $<0.01$, respectively), yet in contrast to my study, they used a single session treatment with carboxymethylcellulose soaked with triamcinolone in contrast to our 8 sessions 1 week apart, and no further follow-up analysis was done at 4 months postintervention.

In a study performed by Bardaranfar et al. [11], they studied the effect on olfactory function posttriamcinolone gelfoam application in comparison with the normal saline control group; their results concluded that application of triamcinolone at the olfactory cleft can boost the effect of surgery in restoring olfactory function. As a matter of fact, their study does not compare to my

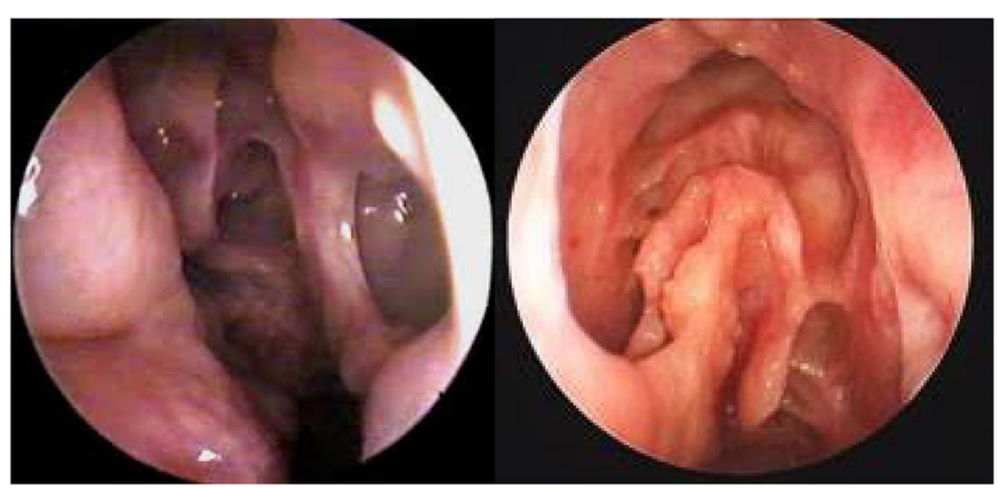

Fig. 2 Post-treatment effect 4 months post-intervention in a sample of patients 
study as they did not impose any endoscopic grading or recurrences, but in fact, they concluded better olfaction outcomes post-FESS surgery through using triamcinolone gelfoam application.

\section{Conclusion}

Triamcinolone-soaked gelfoam packing is an effective and safe method for managing recurrent sinonasal polyposis in patients after FESS. Early intervention contributed to a successful result, and decreased the need for surgery in poor candidates for hypotensive anesthesia and could be used as a sole treatment of recurrent sinonasal polypsis especially in a patient whose general condition could not tolerate invasive procedures or in elderly ages in which invasive procedures are better not resorted or according to patients' preference. Last but not least, patients suffering from recurrent sinonasal polyposis should be given a chance for triamcinolonesoaked gelfoam packing for 8 weeks prior to any invasive procedure.

\section{Further studies}

The patients should be followed up after cessation of treatment for possible recurrence at 6 months and 12 months post-treatment.

\section{Abbreviations}

FESS: Functional endoscopic sinus surgery; SNP: Sinonasal polyposis; NSAI D: Nonsteroidal anti-inflammatory drug

\section{Acknowledgements}

Not applicable.

\section{Author's contributions}

The author declares that he is the single author of this manuscript. The author read and approved the final manuscript.

\section{Funding}

None.

\section{Availability of data and materials}

The datasets generated and analyzed during the current study are not publicly available due participants' confidentiality and personal data issues but are available from the corresponding author on reasonable request.

\section{Declarations}

\section{Ethics approval and consent to participate}

Subjects who agreed to participate in the study were asked to sign a written informed consent. All procedures contributing to this work comply with Good Clinical Practice Guidelines and the ethical principles of the Helwan University Research ethical committee (ENREC) guidelines on human experimentation and with the Helsinki Declaration of 1975, as revised in 2008. The protocol have been revised and approved by the Institution Review Board (IRB no. 5432).

\section{Consent for publication}

Not applicable; images in the manuscript are entirely unidentifiable and there are no details on individuals reported within the manuscript

\section{Competing interests}

The author declares that there are no competing interests.
Received: 2 October 2020 Accepted: 5 April 2021

Published online: 19 May 2021

\section{References}

1. Alobid I, Antón E, Armengot M, Chao J, Colás C, del Cuvillo A et al (2011) SEAIC-SEORL. Consensus document on Nasal Polyposis POLINA Project. J Investig Allergol Clin Immunol. 21(Suppl 1):1-58

2. Armengot M, Garin L, Carda C (2009) Eosinophil degranulation patterns in nasal polyposis: an ultrastructural study. Am J Rhnol Allergy. 23(5):466-470. https://doi.org/10.2500/ajra.2009.23.3357

3. Dufour X, Bedier A, Ferrie JC, Gohler C, Klossek JM (2004) Diffuse nasal polyposis and comorbidity: study of 65 cases treated by endonasal endoscopic surgery. Ann Otolaryngol Chir Cervicofac. 121(5):292-297. https://doi.org/10.1016/S0003-438X(04)95523-5

4. Lanza DC, Kennedy DW (1997) Adult rhinosinusitis defined. Otolaryngol Head Neck Surg. 117(3):S1-S7. https://doi.org/10.1016/S0194-5998(97)70001-9

5. Fokkens W, Lund V, Mullol J; European Position Paper on Rhinosinusitis and Nasal Polyps group (2007) European position paper on rhinosinusitis and nasal polyps 2007. Rhinol Suppl. 20:1-136. PMID: 17844873.

6. Scadding GK, Durham SR, Mirakian R, Jones NS, Drake-Lee AB, Ryan D, Dixon TA, Huber PA, Nasser SM (2008) BSACl guidelines for the management of rhinosinusitis and nasal polyposis. Clin Exp Allergy. 38(2): 260-275. https://doi.org/10.1111/j.1365-2222.2007.02889.x

7. Meltzer EO, Hamilos DL, Hadley JA et al (2006) Rhinosinusitis: developing guidance for clinical trials. J All Clin Immun 118:17-61

8. Lildholdt T, Rundcrantz H, Bende M, Larsen K (1997) Glucocorticoid treatment for nasal polyps. The use of topical budesonide powder, intramuscular betamethasone, and surgical treatment. Arch Otolaryngol Head Neck Surg. 123(6):595-600. https://doi.org/10.1001/archotol.1997.01 900060037006

9. Ryu G, Cho YS, Hong SD, Kim HY, Chung SK, Dhong HJ (2020 May) The effect of triamcinolone-soaked gelfoam in patients with polypoid mucosal change after endoscopic sinus surgery. J Rhinol. 27(1):16-20. https://doi. org/10.18787/jr.2019.00288

10. Pletcher S, Goldberg A (2010) Treatment of recurrent sinonasal polyposis with steroid-infused carboxymethylcellulose foam. Am J Rhinol Allergy 24: 451-453. https://doi.org/10.2500/ajra.2010.24.3523

11. Bardaranfar MH, Ranjbar Z, Dadgarnia MH, Atighechi S, Mirvakili A, Behniafard N, Sadeghi M, Abbaslu F, Baradaranfar A (2014) The effect of an absorbable gelatin dressing impregnated with triamcinolone within the olfactory cleft on polypoid rhinosinusitis smell disorders. Am J Rhinol Allergy. 28(2):172-175. https://doi.org/10.2500/ajra.2014.28.4016 PMID: 24717956

\section{Publisher's Note}

Springer Nature remains neutral with regard to jurisdictional claims in published maps and institutional affiliations.

\section{Submit your manuscript to a SpringerOpen ${ }^{\odot}$ journal and benefit from:}

- Convenient online submission

- Rigorous peer review

- Open access: articles freely available online

High visibility within the field

- Retaining the copyright to your article

Submit your next manuscript at $>$ springeropen.com 